ISSN: 1980-055X

Recebido em: 02/04/2010

Aceito para publicação em: 27/06/2010

\title{
CLIMA E SAÚDE: VARIAÇÕES ATMOSFÉRICAS E ÓBITOS POR DOENÇAS CIRCULATÓRIAS
}

\author{
Pedro Germano Murara ${ }^{17}$ \\ Margarete Cristiane de Costa Trindade Amorim ${ }^{18}$
}

\begin{abstract}
RESUMO
O presente trabalho teve como objetivo investigar os diferentes tipos de tempo atmosférico e suas relações com os registros de óbitos por patologias do aparelho circulatório no município de Presidente Prudente/SP. As doenças circulatórias estão entre as maiores causas de morte no Brasil e desenvolvemse devido a diversos fatores (psicológicos, genéticos, alimentícios, entre outros). Além desses fatores, o fluxo sanguíneo está também relacionado às amplitudes térmicas, que contribuem para a vasodilatação e vasoconstrição do sistema circulatório. Para a realização desta pesquisa foram coletados os dados clínicos de mortalidade no Cartório de Registro Civil, na cidade de Presidente Prudente, referente ao ano de 2007. Durante o mesmo segmento temporal foram coletados dados meteorológicos (temperatura, precipitação, umidade relativa do ar e pressão atmosférica) na Estação Meteorológica da Faculdade de Ciências e Tecnologia - UNESP, campus Presidente Prudente. Foram organizados gráficos com o propósito de comparar os parâmetros climáticos e os registros de mortalidades circulatórias na escala diária. O estudo do clima comparado com a ocorrência de doenças se faz de extrema importância para determinar como os parâmetros climáticos podem influenciar na saúde e na morte das pessoas. Sabe-se que as doenças circulatórias resultam principalmente de fatores de risco (sedentarismo, obesidade, entre outros), além dos parâmetros climáticos. Os resultados apontaram para uma maior concentração de óbitos quando os sistemas atmosféricos estáveis atuavam sobre a área. Os dias com maior número de óbitos por doenças circulatórias estiveram relacionados com períodos de estiagem associados às maiores amplitudes térmicas e dias com baixos valores de umidade relativa do ar, demonstrando maior concentração dos agravos quando os sistemas atmosféricos estáveis atuavam sobre a área ou quando os mesmos sofriam um aquecimento basal.
\end{abstract}

Palavras-chave: Climatologia geográfica; Clima e saúde; Ritmo climático; Doenças circulatórias;

\footnotetext{
${ }^{17}$ Mestrando do PPGG da Universidade Federal de Santa Catarina (UFSC), pmurara@gmail.com

${ }^{18}$ Profa. Dra. Departamento de Geografia da Faculdade de Ciências e Tecnologia (FCT)-Universidade Estadual Paulista (UNESP), mccta@fct.unesp.br
} 
ABSTRACT

This study aimed to investigate the different types of weather and its relations with the records of deaths from diseases of the circulatory system in the city of Presidente Prudente / SP. Circulatory diseases are one of the main causes of death in Brazil and develop from different factors (psychological, genetic, food, and others.). Besides these factors, blood flow is also related to temperature variations, which contribute to vasodilatation and vasoconstriction of the circulatory system. For this research, clinical data of mortality in the Civil Registry Office in the city of Presidente Prudente were collected, for the year 2007. During the same weather segment were collected meteorological data (temperature, precipitation, relative humidity and atmospheric pressure) at the Meteorological Station of the Faculty of Science and Technology - UNESP, Presidente Prudente College. Graphs were organized with the purpose of comparing the climatic parameters and records of circulatory mortality in the daily scale. The climate study compared to the occurrence of disease becomes of paramount importance to determine how climatic parameters can influence the health and death of people. It is known that circulatory diseases mainly result from risk factors (sedentary lifestyle, obesity, among others), in addition to climatic parameters. Results showed a higher concentration of deaths when the stable weather systems worked on the zone. The days with the highest number of deaths from circulatory diseases were related to periods of drought associated with the higher temperature range, and days with low values of relative humidity, showing higher concentration of injuries when the stable weather systems worked over the area or when they suffered a basal heating.

Key words: Climatology geographical, climate and health; Rhythm climate; Cardiovascular disease;

\section{INTRODUÇÃo}

Determinadas doenças manifestam-se, surgem ou desenvolvem-se devido às variações (naturais ou antrópicas) periódicas dos elementos climáticos. O clima e os diferentes tipos de tempo (ondas de calor, períodos de estiagem, variações súbitas das temperaturas), são entendidos como um fator ambiental que influencia no organismo humano, não com um caráter determinista, mas como um elemento que pode contribuir de maneira benéfica ou maléfica para a saúde humana.

Lacaz (1972, p. 24) destaca que "os elementos climáticos interferem de modo marcante no aparecimento e na manutenção de determinadas doenças". Ayoade (2007, p. 290) afirma que "temperaturas extremamente altas provocam a incidência de choques térmicos, exaustão e cãibras pelo calor". Por outro lado, temperaturas extremamente baixas causam doenças como artrites, sinusites e enrijecimento de juntas. O ar muito seco prejudica ou diminui o vigor físico e a disposição para o trabalho mental.

As DAC (doenças do aparelho circulatório) estão entre as principais causas de morte no Brasil, devido às complicações que elas desenvolvem (derrame cerebral, edema agudo do pulmão, infarto do miocárdio, entre 
outras). A hipertensão arterial, por exemplo, uma patologia circulatória, provoca o aumento do fluxo sanguíneo e a sua variação depende de vários fatores, dentre eles, as amplitudes térmicas, que contribuem para a vasodilatação e vasoconstrição do sistema circulatório.

Conforme consulta realizada no banco de dados do DATASUS, as doenças do aparelho circulatório são responsáveis pelo maior número de mortes no Brasil. Na tabela 1, apresentam-se os registros de mortalidade no município de Presidente Prudente para os anos de 2000 a 2007. Nessa tabela, verifica-se expressivo o número de casos de pacientes acometidos por agravos circulatórios, revelando também as DAC como primeira causa de mortalidade no município paulista.

Tabela 01 - Mortalidade hospitalar no município de Presidente Prudente - SP.

\begin{tabular}{|c|c|c|c|c|c|c|c|c|c|}
\hline CID-10 - Presidente Prudente & \multicolumn{8}{|c|}{ ANO DE OCORRÊNCIA } & \multirow[b]{2}{*}{ Total } \\
\hline Capítulo & 2000 & 2001 & 2002 & 2003 & 2004 & 2005 & 2006 & 2007 & \\
\hline I. Algumas doenças infecciosas e parasitárias & 29 & 33 & 47 & 34 & 33 & 45 & 99 & 106 & 426 \\
\hline II. Neoplasias (tumores) & 17 & 17 & 30 & 37 & 50 & 73 & 70 & 81 & 375 \\
\hline III. Doenças sangue órgãos hemat e transt imunitár & 8 & 11 & 14 & 5 & 2 & 5 & 5 & 4 & 54 \\
\hline IV. Doenças endócrinas nutricionais e metabólicas & 21 & 18 & 38 & 22 & 23 & 15 & 43 & 16 & 196 \\
\hline V. Transtornos mentais e comportamentais & 9 & 15 & 16 & 13 & 18 & 4 & 8 & 7 & 90 \\
\hline VI. Doenças do sistema nervoso & 14 & 8 & 4 & 9 & 8 & 13 & 29 & 6 & 91 \\
\hline IX. Doenças do aparelho circulatório & 118 & 138 & 153 & 123 & 118 & 93 & 137 & 130 & 1010 \\
\hline X. Doenças do aparelho respiratório & 95 & 106 & 136 & 141 & 115 & 55 & 102 & 93 & 843 \\
\hline XI. Doenças do aparelho & 57 & 72 & 65 & 53 & 40 & 50 & 60 & 32 & 429 \\
\hline XII. Doenças d & 2 & 2 & 2 & 2 & 0 & 2 & 0 & 0 & 10 \\
\hline XIII.Doenças sist osteomus & 7 & 8 & 5 & 7 & 7 & 5 & 4 & 4 & 47 \\
\hline XIV. Doenças do aparelho geniturinário & 15 & 20 & 12 & 15 & 23 & 13 & 14 & 11 & 123 \\
\hline XV. Gravidez parto e puerpério & 0 & 1 & 1 & 1 & 0 & 0 & 2 & 0 & 5 \\
\hline $\begin{array}{l}\text { XVI. Algumas afec originadas no período perinatal } \\
\text { XVII. Malf cong deformid e anomalias }\end{array}$ & 19 & 17 & 15 & 13 & 22 & 6 & 8 & 13 & 113 \\
\hline cromossômicas & 7 & 4 & 3 & 4 & 6 & 3 & 4 & 9 & 40 \\
\hline XVIII. Sint sinais e achad & 13 & 18 & 24 & 19 & 10 & 10 & 23 & 19 & 136 \\
\hline XIX. Lesões enven e alg out conseq causas externas & 31 & 28 & 35 & 19 & 35 & 29 & 29 & 47 & 253 \\
\hline XX. Causas externas de morbidade e mortalidade & 1 & 0 & 0 & 0 & 0 & 0 & 0 & 0 & 1 \\
\hline XXI. Contatos com serviços de saúde & 0 & 3 & 0 & 0 & 1 & 1 & 0 & 3 & 8 \\
\hline Total & 463 & 519 & 600 & 517 & 511 & 422 & 637 & 581 & 4250 \\
\hline
\end{tabular}

Fontes: Sistema de Informações Hospitalares do SUS (SIH), Departamento de Informática do SUS (DATASUS), 2000 a 2007.

Adaptação: Murara, 2008.

O clima atua na manifestação de determinados agravos à saúde, tendo seus elementos - temperatura do ar, umidade, precipitação, pressão atmosférica e ventos - interferência no bem estar dos indivíduos. Esses elementos não são os únicos responsáveis pelo desencadeamento de enfermidades; todavia, quando vinculados às características físicas, psicológicas e culturais, ou seja, fatores de riscos ligados ao estilo de vida (dieta e obesidade, exercícios físicos, tabagismo, níveis de colesterol, fatores de coagulação e suscetibilidade), resultam como mais um contribuinte para o agravamento de determinadas enfermidades.

Estudos que relacionam a influência dos elementos climáticos sobre as doenças circulatórias (PITTON e DOMINGOS, 2004; CASTILHO, 2006; PASCOALINO, 2008) constataram uma tendência sazonal no desencadeamento das enfermidades, sendo estas mais ocorrentes no período invernal, com prevalência de extremos térmicos acentuados e predomínio de tempo seco e estável. 
O município de Presidente Prudente situa-se no extremo oeste do Estado de São Paulo, a 587 km da capital e ocupa uma área de 562 km² (Figura 1). Possui aproximadamente 203.164 habitantes dos quais $98,1 \%$ residem na área urbana (IBGE, 2006). Está inserido no planalto ocidental paulista, com colinas suavemente onduladas e com altitudes entre $375 \mathrm{~m}$ e $480 \mathrm{~m}$ acima do nível do mar. Localiza-se em uma área de transição climática, influenciado pela alternância dos sistemas tropicais e polares, mas dominado por massas de ar Tropical Marítima. (AMORIM, 2000).

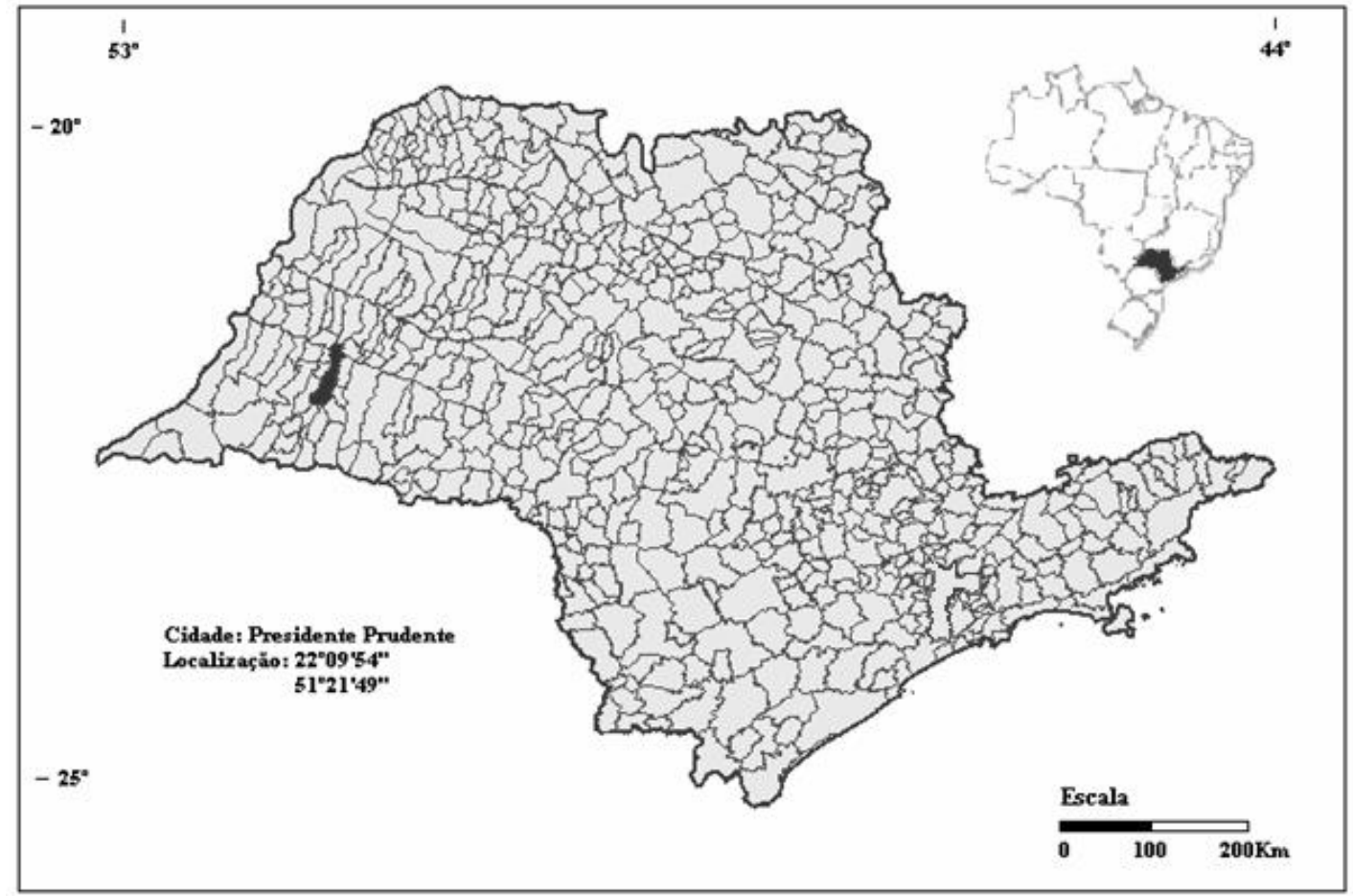

Figura 01 - Localização do município de Presidente Prudente.

Fonte: IBGE, 2007.

Elaboração: Murara, 2008.

A participação da FPA (Frente Polar Atlântica) e da ZCAS (Zona de Convergência do Atlântico Sul) na gênese das chuvas é significativa. A região recebe invasões e perturbações frontais, mesmo na primavera-verão, quando as chuvas são mais frequentes e intensas. Verificam-se durante o ano duas estações bem definidas na região; uma estação seca, de tempo estável (outonoinverno), e outra quente e úmida com chuvas mais frequentes e intensas (primavera-verão). A presença de sistemas estabilizadores de tempo no outono e no inverno provoca uma redução das chuvas que culmina em um período seco, no qual a precipitação se torna quase que exclusivamente ligada à ação frontal (BARRIOS \& SANT' ANNA NETO, 1996; AMORIM, 2000).

Nos meses de verão, quando a região é dominada pelas massas de ar quente, registram-se os valores mais baixos de pressão atmosférica e, no inverno, ocorre o oposto. A média anual da pressão atmosférica em Presidente Prudente é de $1013 \mathrm{mb}$, sendo a sua variação mensal muito pequena, com oscilação entre $1012 \mathrm{mb}$ e $1015 \mathrm{mb}$. A temperatura tem média anual de $23,1^{\circ} \mathrm{C}$, com variação entre $21,9^{\circ} \mathrm{C}$ e $24^{\circ} \mathrm{C}$. A continentalidade é responsável pelas 82 
elevadas temperaturas médias anuais. Em geral, anos mais chuvosos são menos quentes e os anos mais secos apresentam maior temperatura (BARRIOS \& SANT'ANNA NETO, 1996).

Ainda com relação à continentalidade, a cidade de Presidente Prudente apresenta umidade relativa do ar não muito elevada, variando, em média, entre $60 \%$ a $70 \%$, ao longo do ano. Quanto à variação sazonal, de dezembro a março, a umidade fica em torno dos $70 \%$, diminuindo para menos de $60 \%$ nos meses de julho, agosto e setembro. Desta forma, a média anual de precipitação $1250 \mathrm{~mm}$, concentra-se nos meses de outubro a março com 73\% do total anual, restando apenas $27 \%$ que se precipitam nos seis meses mais secos. O trimestre de dezembro a fevereiro é responsável por quase $40 \%$ do total anual (mais de $500 \mathrm{~mm}$ ). A estiagem que tem início em julho se estende até setembro com cerca de $10 \%$ do total anual (BARRIOS \& SANT 'ANNA NETO, 1996).

Nesse contexto, o presente trabalho teve como objetivo investigar as características do tempo atmosférico e a ocorrência de óbitos decorrentes de enfermidades circulatórias que se desenvolvem e ou se agravam sob determinados tipos de tempo atmosférico nos residentes urbanos de Presidente Prudente/SP, durante o ano de 2007.

\section{MATERIAL E MÉTODOS}

Para compreender as possíveis relações entre as características do tempo atmosférico com a ocorrência de óbitos por doenças circulatórias nos residentes de Presidente Prudente/SP, foram coletados dados diários dos elementos climáticos e dos registros de óbitos por doenças do aparelho circulatório do ano de 2007.

Os dados climáticos (temperatura, precipitação, umidade relativa do ar, pressão atmosférica, direção e velocidade do vento), foram coletados na Estação Meteorológica da FCT - UNESP, campus Presidente Prudente.

Os dados clínicos de mortalidade (do mesmo segmento temporal) foram coletados junto ao Cartório de Registro Civil de Presidente Prudente. Foram considerados os óbitos classificados segundo o IX Capítulo de Doenças do Aparelho Circulatório (DAC) (Cód. I00-I99) da 10a Revisão da Classificação Internacional de Doenças (10a CID).

Foram analisados diariamente os óbitos por doenças do aparelho circulatório na cidade de Presidente Prudente/SP, durante o período de 01 de janeiro a 31 de dezembro de 2007. Os dados foram organizados a partir da planilha eletrônica do $\operatorname{EXCEL}^{19}$, para a elaboração dos gráficos de análise rítmica, baseada na proposta de Monteiro (1971), a fim de analisar a variação dos elementos climáticos de acordo com os sistemas atmosféricos atuantes, com o propósito de comparar os parâmetros climáticos e os óbitos por enfermidades do aparelho circulatório.

A utilização do software CorelDraw ${ }^{20}$ reuniu os gráficos elaborados anteriormente (em planilha eletrônica) de maneira a facilitar a análise e compreensão dos diferentes tipos de tempo em escala diária.

Para auxiliar na identificação dos diferentes tipos de tempo atmosféricos foram utilizadas imagens de satélite e boletins climáticos (Climanálise)

\footnotetext{
19 EXCEL - é a marca registrada da Microsoft Corporation.

${ }^{20}$ Coreldraw - é a marca registrada da Corel Corporation.
} 
disponíveis no site do CPTEC/INPE e ainda, cartas sinóticas, nível de superfície, diárias e na escala da América Latina no site da Marinha do Brasil, além dos dados meteorológicos de superfície.

\section{RESULTADOS}

A análise dos óbitos por doenças circulatórias e as condições atmosféricas ocorridas em Presidente Prudente no transcorrer do ano de 2007, demonstrou maior concentração dos agravos quando os sistemas atmosféricos estáveis (Massa Tropical Atlântica - mTa e Massa Polar Atlântica - mPa) atuavam sobre a área ou quando os mesmos sofriam um aquecimento basal (Massa Tropical Atlântica Continentalizada - mTac e Massa Polar Atlântica Velha ou tropicalizada - mPav) resultando em maior quantidade de registros de óbitos por doenças do aparelho circulatório (Figuras 2 e 3 ).

Como exemplo, verificou-se no mês de março (Figura 2), casos isolados de mortes por doenças circulatórias e aumento nos registros de óbitos depois da atuação dos sistemas estáveis (mTa e mTac).

É preciso ressaltar que, os óbitos por doenças circulatórias não ocorrem imediatamente na atuação do sistema atmosférico, já que o agravamento da doença se dá em dias, posteriormente há a internação e salvo alguns casos, o óbito. É preciso levar em conta essa defasagem de dias. Outros, no entanto, são vítimas diretas de ataques fulminantes da enfermidade resultando diretamente em morte do indivíduo.

Nesse contexto, o final do mês de março foi caracterizado pela mTa que atuou até o início do mês de abril. Como consequência a esse sistema (quente e seco), observou-se que a quantidade de óbitos por problemas circulatórios aumentou no mês de abril (Figura 3 ).

No início do inverno, no mês de junho de 2007, quando a área estava sob o domínio de sistemas polares e tropicais, o número óbitos também aumentou (Figura 4).

Através da análise da precipitação diária e óbitos por doenças circulatórias no ano de 2007, notou-se que nos dias de estiagem (ou com precipitações isoladas), o número de óbitos aumentou. Essa situação pode ser observada na Figura 4.

Com relação às temperaturas do ar, ficou evidenciado que os dias de maior amplitude térmica resultaram em aumento no número de óbitos por doenças circulatórias. No inverno, período do ano em que se apresentam as maiores amplitudes térmicas (chegando a ser registrada amplitude térmica máxima de $17,4{ }^{\circ} \mathrm{C}$ no dia 01 de junho) foi observado, também, os maior número de óbitos (Figura 4).

A relação entre a umidade relativa do ar e os óbitos por doenças circulatórias, evidenciou que não houve aumento de óbitos nos dias que apresentaram os maiores valores de umidade relativa do ar (Figura 5), ao passo que nos dias em que foram registrados valores baixos (50\%) de umidade relativa do ar, a incidência de casos de óbitos aumentou. 


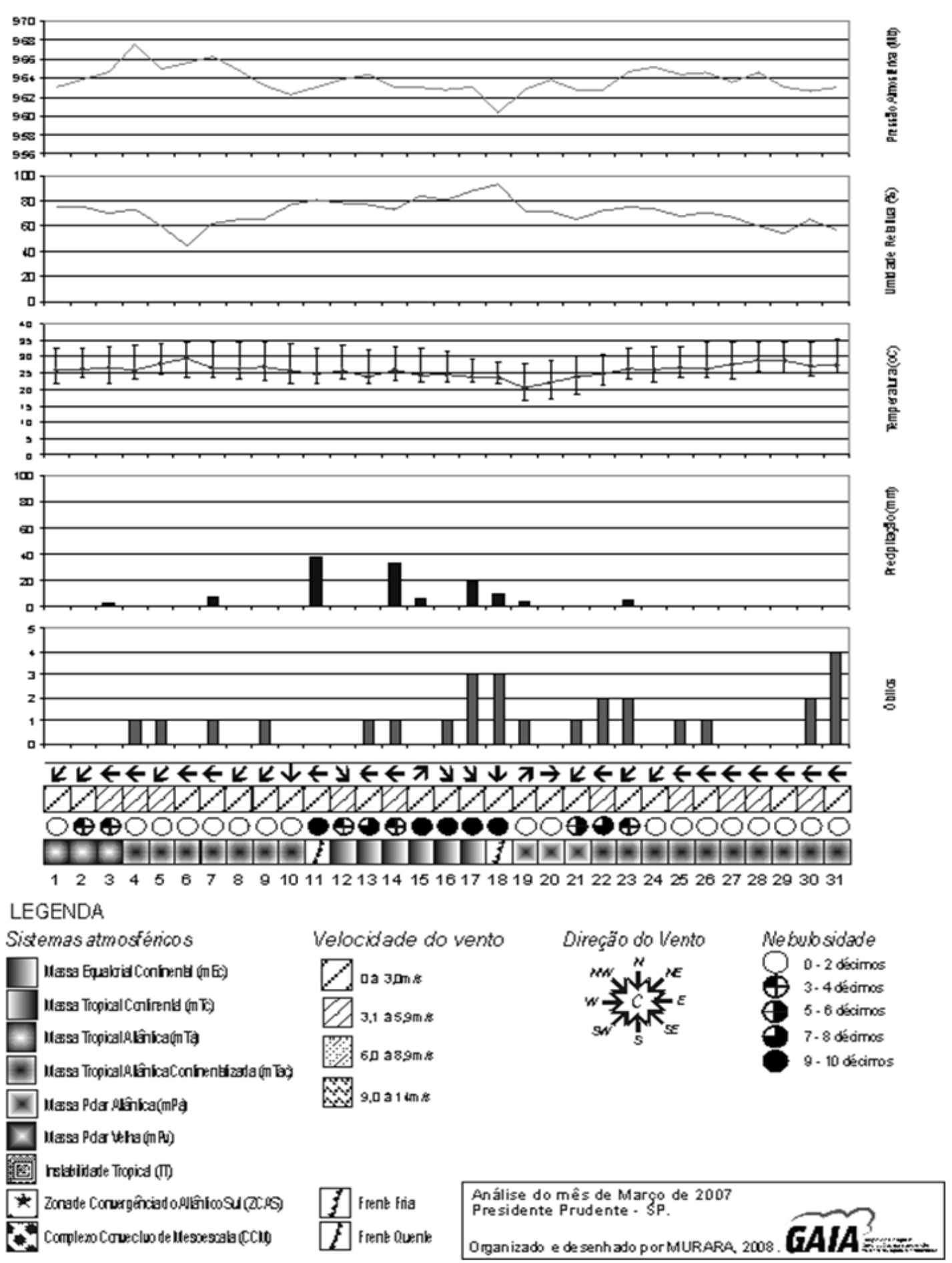

Figura 02 - Elementos do clima e óbitos por doenças do aparelho circulatório, em Presidente Prudente, março de 2007.

Fonte: Estação Meteorológica de Presidente Prudente e Cartório de Registro Civil, 2007.

Elaboração: Murara, 2009. 


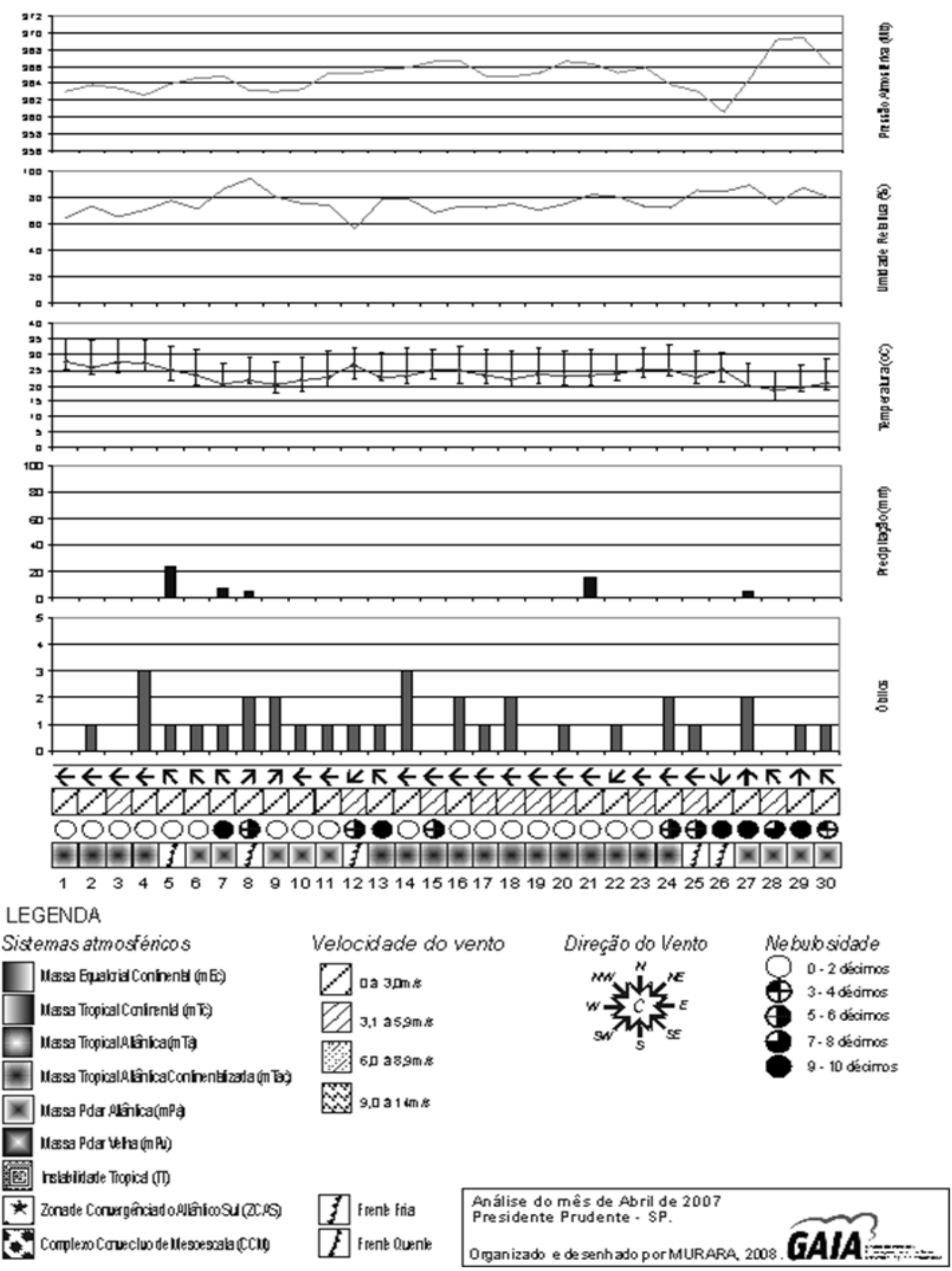

Figura 03 - Elementos do clima e óbitos por doenças do aparelho circulatório, em Presidente Prudente, abril de 2007.

Fonte: Estação Meteorológica de Presidente Prudente e Cartório de Registro Civil, 2007.

Elaboração: Murara, 2009. 


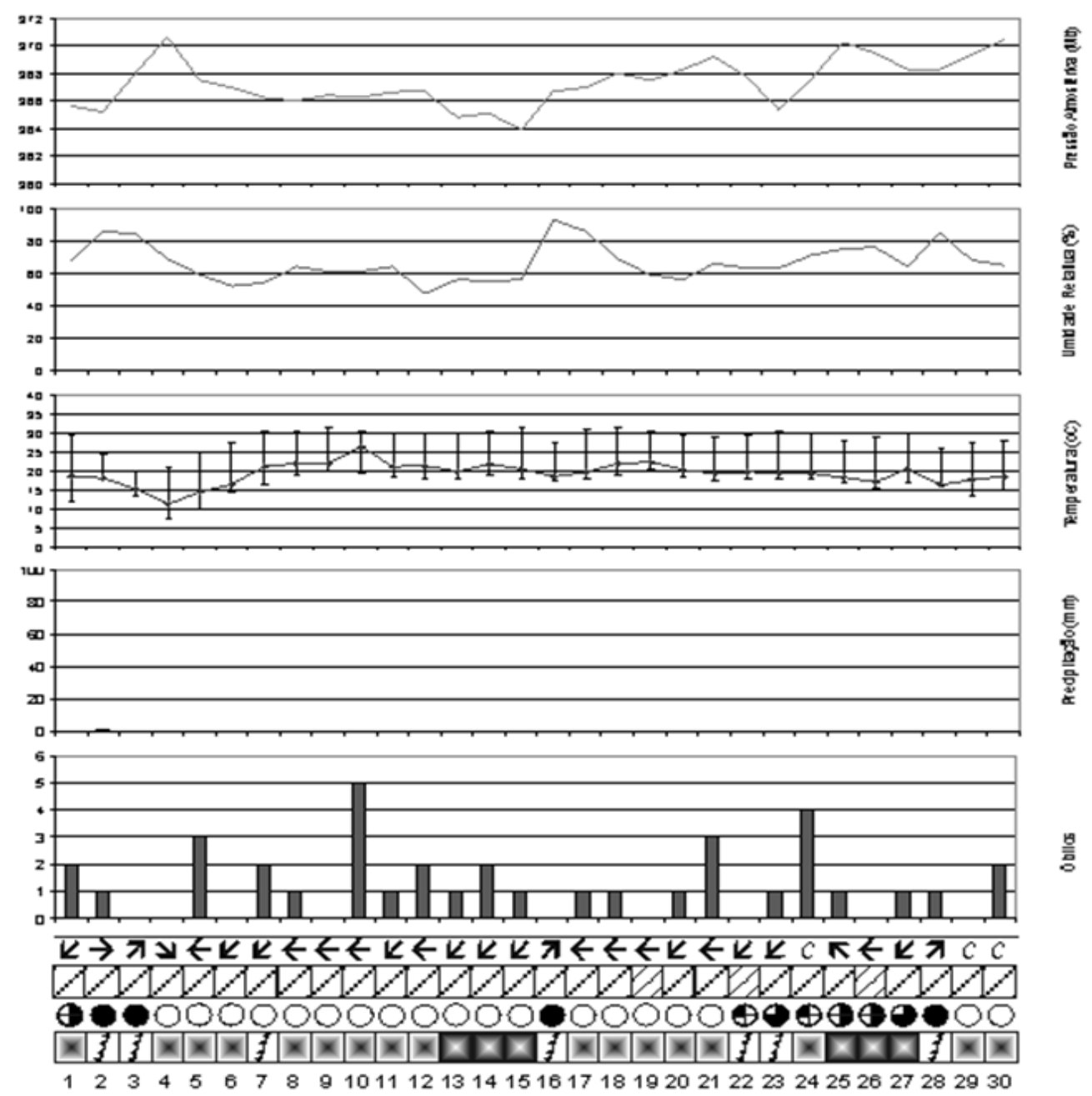

LEGENDA

\section{Sistemas atmosfénicos}

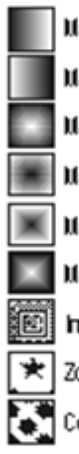

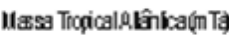

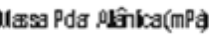

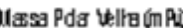

relatilidale Tropicd (II)
Iasa Equabia Conirent ún

Uæsa Tropical Conirenta (mT)

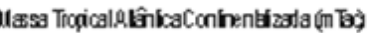

Zonade Corưgîncia do.

Complexo Conue civo de llessesala (ccit)
Velocidade do vento

$\angle$ oa $3 \mathrm{~mm} s$

V., as,sm:

6D as, $9 \mathrm{~m}: 8$

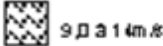

Direçäo do Vento

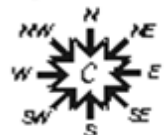

Nebubsidade

(Q) 0.2 dscimos

क 3.4 dédrmos

(f) 5.6 décimos

7.8 dédimos

9. 10 décirros

Figura 04 - Elementos do clima e óbitos por doencas do aparelho circulatório, em Presidente Prudente, junho de 2007.

Fonte: Estação Meteorológica de Presidente Prudente e Cartório de Registro Civil, 2007.

Elaboração: Murara, 2009. 


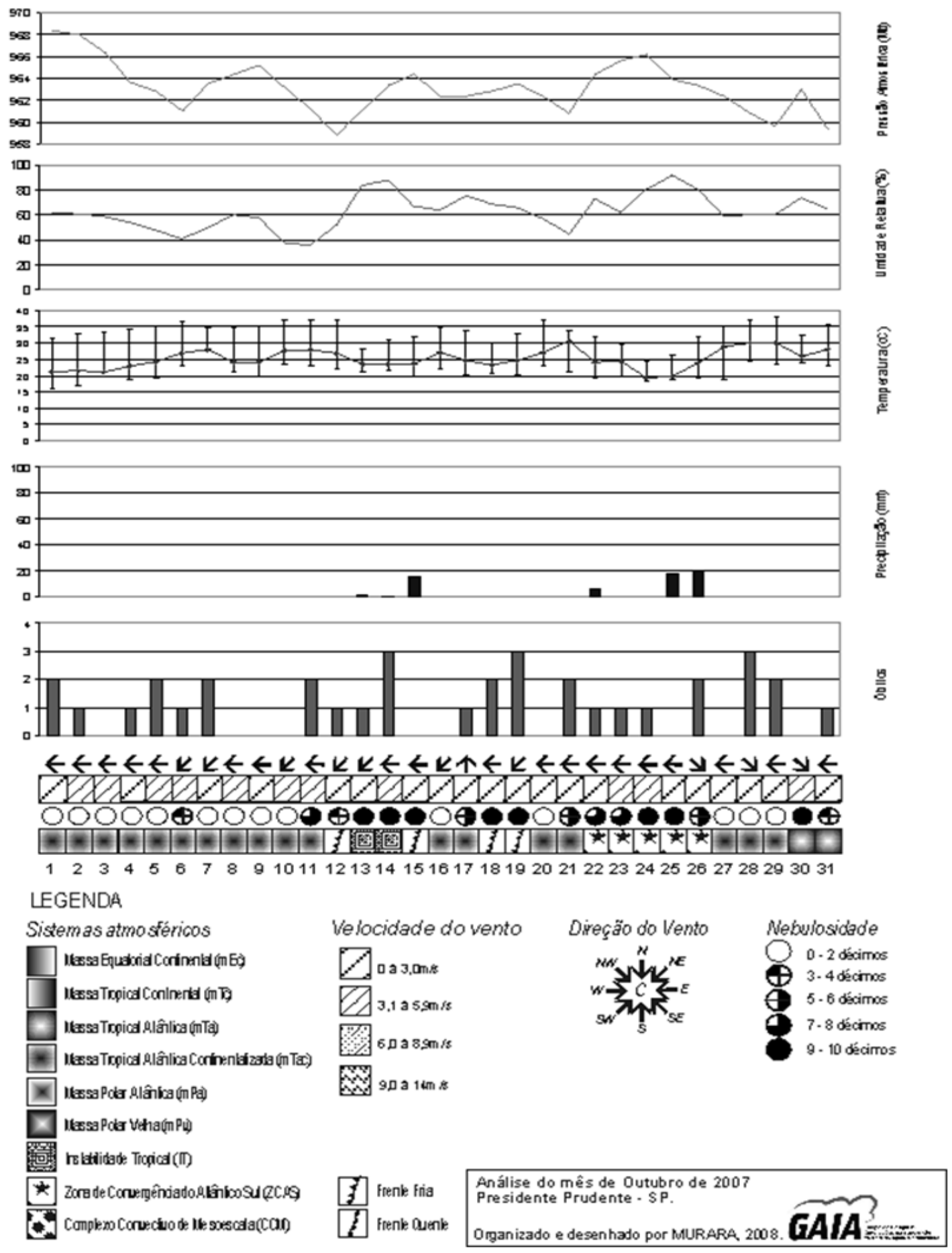

Figura 05 - Elementos do clima e óbitos por doenças do aparelho circulatório, em Presidente Prudente, outubro de 2007.

Fonte: Estação Meteorológica de Presidente Prudente e Cartório de Registro Civil, 2007.

Elaboração: Murara, 2009. 
A partir do exposto, foi possível a elaboração da Tabela 02 que relacionou o número de dias em que diferentes sistemas atmosféricos atuaram nos meses de 2007 no município de Presidente Prudente. A partir da identificação dos diferentes tipos de tempo, acrescentaram-se os óbitos por DAC (doenças do aparelho circulatório).

Tabela 02 - Número de dias com óbitos por doenças do aparelho circulatório e a atuação de sistemas atmosféricos durante o ano de 2007, em Presidente Prudente.

\begin{tabular}{l|c|c|c|c|c|c|c|c|}
\hline Meses & óbitos & mTa e mTac & mPa e mPv & mEc & mTc & ZCAS & FF & IT \\
\hline JAN & 14 & $*$ & 8 & 3 & $*$ & 14 & 4 & 2 \\
FEV & 30 & 18 & $*$ & $*$ & $*$ & 10 & $*$ & $*$ \\
MAR & 27 & 20 & 3 & 6 & $*$ & $*$ & 2 & $*$ \\
ABR & 32 & 16 & 9 & $*$ & $*$ & $*$ & 5 & $*$ \\
MAI & 25 & $*$ & 24 & $*$ & $*$ & $*$ & 7 & $*$ \\
JUN & 37 & $*$ & 23 & $*$ & $*$ & $*$ & 7 & $*$ \\
JUL & 27 & $*$ & 23 & $*$ & $*$ & $*$ & 8 & $*$ \\
AGO & 25 & 2 & 24 & $*$ & $*$ & $*$ & 5 & $*$ \\
SET & 32 & 14 & 11 & $*$ & $*$ & $*$ & 5 & $*$ \\
OUT & 35 & 20 & $*$ & $*$ & $*$ & 5 & 4 & 2 \\
NOV & 18 & 4 & 4 & $*$ & 4 & 10 & 8 & $*$ \\
DEZ & 28 & 7 & 4 & 2 & 6 & 7 & 5 & $*$
\end{tabular}

* Não atuação do sistema

Elaboração: Murara, 2009.

Nos meses em que o maior número de dias esteve sob a atuação dos sistemas estáveis ( $\mathrm{mTa}, \mathrm{mTac}, \mathrm{mPa}$ e $\mathrm{mPv}$ ), o número de registros de óbitos por DAC foi maior. Cabe lembrar que, a presença de sistemas estabilizadores de tempo no outono e no inverno provoca a redução das chuvas que culmina em um período seco (BARRIOS \& SANT`ANNA NETO, 1996). Dessa forma, nos períodos com estiagens (ou com precipitações isoladas) foram registrados os maiores totais de óbitos. Esse fato foi observado nos meses de maio, junho, julho e agosto, com predomínio da atuação da Massa Polar Atlântica (mPa) e da Massa Polar Velha (mPv), que se caracterizaram por baixas temperaturas e amplitudes térmicas elevadas (Figura 4) e proporcionaram dias estáveis, que resultaram no aumento do número de registros de óbitos por doenças circulatórias (Tabela 02).

O corpo humano saudável apresenta maior eficiência a uma temperatura central de $37^{\circ} \mathrm{C}$ (equilíbrio térmico). As variações dos tipos de tempo influenciam a temperatura corporal, que por sua vez ativam os mecanismos naturais (homeostáticos) humanos para o controle do equilíbrio entre o meio externo e o organismo interno. O organismo humano responde a esse processo ativando o funcionamento da vasoconstrição e vasodilatação, sobrecarga ou menor fluxo dos vasos sanguíneos e do coração. (TORTORA, 2000). Dessa forma, normalmente ocorre o aumento da tensão arterial, podendo resultar na maior incidência de doenças circulatórias.

Situações extremas, como ondas de calor no verão e de frio no inverno, afetam a saúde e o bem estar de diversas formas. A combinação de temperaturas baixas e ventos podem fazer com que a temperatura do ar seja significativamente mais baixa, podendo conduzir mais facilmente à hipotermia 
(temperatura corporal abaixo de $35^{\circ} \mathrm{C}$ ), que é produzida pelo frio excessivo. Nesse caso, o cérebro perde a capacidade de regular a temperatura corporal. A frequência cardíaca cai, a respiração fica mais lenta e os vasos se contraem, aumentando a pressão sanguínea, podendo ocorrer perda de consciência (desmaio), congelamento das extremidades e parada cardíaca (JACKSON, 2000).

Não há dúvidas de que os fatores individuais: tabagismo, dieta sem orientação, stress dentro das atividades diárias, o álcool, hereditariedade, entre outros, exerçam influências nas doenças cardiovasculares. No entanto, os parâmetros climáticos, tais como: precipitação, pressão atmosférica, temperatura, umidade relativa do ar, direção e velocidade dos ventos, quando combinados a diferentes tipos de tempos atmosféricos, contribuem para o desencadeamento das enfermidades cardiovasculares, sendo assim mais um fator a ser analisado como influente nas doenças e entendido não como responsável por elas.

\section{CONCLUSÕES}

Este estudou evidenciou que as características do tempo atmosférico podem exercer influência nos óbitos por doenças do aparelho circulatório. Por se tratar de um estudo preliminar, se faz necessária novas abordagens acerca da relação existente entre os elementos climáticos e os registros de óbitos por doenças do aparelho circulatório, assim como dos registros de morbidade.

Durante o ano em análise, ficou claro que os parâmetros climáticos exerceram uma influência natural na incidência de óbitos por doenças circulatórias, demonstrando maior concentração dos agravos quando os sistemas atmosféricos estáveis (Massa Tropical Atlântica e Massa Polar Atlântica) atuavam sobre a área ou quando os mesmos sofriam um aquecimento basal (Massa Tropical Atlântica Continentalizada e Massa Polar Velha).

Os dias com maior número de óbitos por doenças circulatórias estiveram relacionados com períodos de estiagens associados às maiores amplitudes térmicas, ou ainda, dias com baixos valores de umidade relativa do ar.

Atualmente se discutem as ações humanas na alteração dos climas regionais; no entanto, pequenas ações podem ser realizadas de modo a atenuar as influências do clima local, tais como, minimizar as amplitudes térmicas, o aumento da umidade relativa do ar e a poluição local, através do aumento de áreas verdes (praças, jardins, parques etc.) ou ainda, incentivar a criação de espelhos d'água e privilegiar a canalização de córregos a céu aberto e não mais as canalizações fechadas. Tais medidas poderão aumentar a área de infiltração e como resultado aumentar a evapotranspiração (quando da presença de vegetação), minimizando as temperaturas locais.

Ressaltam-se ainda, as preocupações com os padrões construtivos, as características de moradia, como ventilação, iluminação, insolação, umidade relativa e temperatura ambiente (interna das edificações). Todos esses fatores influenciam direta ou indiretamente na saúde humana, no conforto térmico e, por conseguinte, nas doenças do aparelho circulatório.

Salienta-se nesse contexto, a necessidade de realização de estudos dessa natureza, trabalhando-se a correlação de outros parâmetros climáticos e em escalas mais detalhadas, de modo que se proporcione a observação das 
influências das sazonalidades climáticas nas incidências de internações e óbitos por doenças do aparelho circulatório, a fim de contribuir com planejamentos públicos menos voltados para o tratamento dessas enfermidades, mas direcionados para os processos preventivos, visando a cuidar da saúde e não da enfermidade.

\section{REFERÊNCIAS BIBLIOGRÁFICAS}

AMORIM, M.C.C.T. O clima urbano de Presidente Prudente/SP. São Paulo, 2000. 374p. Tese (Doutorado em Geografia) - Faculdade de Filosofia, Letras e Ciências Humanas, Universidade de São Paulo.

AYOADE, J.O. Introdução á climatologia para os trópicos. 12a Edição. Rio de Janeiro: Bertrand Brasil, 2007.

BARRIOS, N.A.Z.; SANT'ANNA NETO, J.L. A circulação atmosférica no extremo oeste paulista. Boletim climatológico, v.1, n.1, p.8-9, março 1996.

CASTILHO, F.J.V. Abordagem geográfica do clima urbano e das enfermidades em São José do Rio Preto/SP. Rio Claro, 2006. Dissertação (Mestrado em Geografia) - Instituto de Geociências e Ciências Exatas, Universidade Estadual Paulista.

DOMINGOS, A. E. Alterações climáticas e doenças cardiovasculares no município de Santa Gertrudes - SP. Rio Claro. 2001. Monografia (Bacharel em Geografia) - Instituto de Geociências e Ciências Exatas, Universidade Estadual Paulista.

Instituto Brasileiro de Geografia e Estatística. IBGE. Cidades. Acesso em: 28 de out de 2007. Disponível em: http://www.ibge.org.br/cidades.htm

JACKSON, G. Tudo sobre doenças cardíacas. São Paulo, Org. Andrei, 2000.

Ministério de Saúde. Departamento de Informática do Sistema Único de Saúde. DATASUS. Acesso em: 05 de ago de 2007. Disponível em: http://www.datasus.org.br

MONTEIRO, C. A. F. Análise rítmica em climatologia - problemas da atualidade climática em São Paulo e achegas para um programa de trabalho. In: Climatologia 1. São Paulo: IGEOG/USP, 1971.

A dinâmica e as chuvas no Estado de São Paulo. São Paulo: IGEOG/USP, 1973 (Estudos Geográficos sob forma de Atlas).

LACAZ, C. S. Introdução à Geografia Médica do Brasil. São Paulo: EDUSP, 1972. 
PITTON, S. E. e DOMINGOS, A. E. Tempos e doenças: efeitos dos parâmetros climáticos nas crises hipertensivas nos moradores de Santa Gertrudes - SP. In. Estudos Geográficos. Rio Claro, vol. 02, nº 01, p. 75-86, 2004.

SOUZA, C.G. de. A influência do ritmo climático na morbidade respiratória em ambientes urbanos. Presidente Prudente, 2007. 179p. Dissertação (Mestrado em Geografia) - Faculdade de Ciências e Tecnologia, Universidade Estadual Paulista.

TORTORA, G. J. O Sistema Circulatório. In: Corpo Humano: fundamentos de anatomia e fisiologia. Porto Alegre: Armed Editora, 2000.

VICENTE, A.K. Conforto térmico na cidade de Presidente Prudente. Presidente Prudente, 2001. 60p. Monografia (Bacharel em Geografia) Faculdade de Ciências e Tecnologia, Universidade Estadual Paulista. 